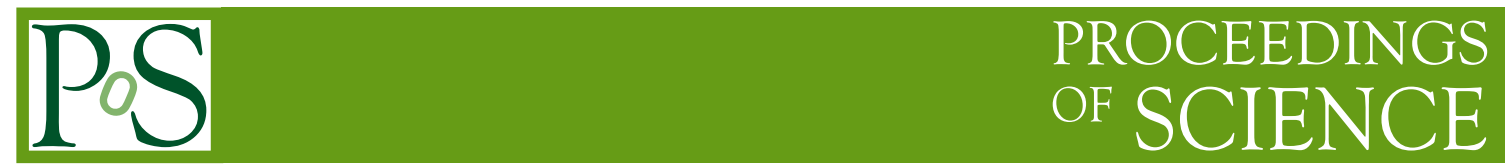

\title{
Measurements of the CKM angle $\gamma$ at LHCb
}

\section{Mark Whitehead*广}

RWTH Aachen University

E-mail: mwhitehe@cern.ch

The latest time-integrated $\gamma$ measurement using $B^{ \pm} \rightarrow D K^{ \pm}$with $D \rightarrow K_{S}^{0} \pi^{+} \pi^{-}$and $D \rightarrow$ $K_{S}^{0} K^{+} K^{-}$decays using LHCb Run 2 data is presented, and gives the most precise single determination of $\gamma$ to date. This, and other new results, are including in the latest LHCb $\gamma$ combination to give $\gamma=\left(74.0_{-5.8}^{+5.0}\right)^{\circ}$, the most precise measurement of $\gamma$ from a single experiment.

The 39th International Conference on High Energy Physics (ICHEP2018)

4-11 July, 2018

Seoul, Korea

* Speaker.

${ }^{\dagger}$ On behalf of the LHCb collaboration. 


\section{Introduction}

The CKM angle $\gamma$ is one of the least well known parameters of the unitarity triangle. Measuring $\gamma$ precisely is a key goal of flavour physics because it is a standard candle measurement of $C P$ violation, since it can be determined from tree-level decays where new physics effects are expected to be small. Additionally, theoretical uncertainties associated with measuring $\gamma$ are negligible, so experiments must continue to improve precision. The current world average from HFLAV is $\left(73.5_{-5.1}^{+4.2}\right)^{\circ}[1]$, which is dominated by the $2017 \mathrm{LHCb}$ gamma combination result $\left(76.8_{-5.7}^{+5.1}\right)^{\circ}$ [2].

\section{LHCb model independent GGSZ analysis with $B^{ \pm} \rightarrow D K^{ \pm}$decays}

The GGSZ method, named after the authors of Ref. [3], uses decays of neutral $D$ mesons to multibody final states. The decay $B^{+} \rightarrow D K^{+}$proceeds via favoured $b \rightarrow c$ or colour and CKM suppressed $b \rightarrow u$ transitions. The weak phase difference between these amplitudes is $\gamma$, and $r_{B}$ and $\delta_{B}$ are the ratio of magnitudes and the strong phase difference, respectively. Sensitivity to $\gamma$ comes from the interference between these amplitudes when the neutral $D$ meson decays to a final state common to $D^{0}$ and $\bar{D}^{0}$.

The GGSZ method uses the distribution of candidates over the $D$ Dalitz plot for $B^{+}$and $B^{-}$ decays, and requires knowledge of the strong phase variation across the Dalitz plot, which can either be taken from external measurements (model independent) or amplitude fits (model dependent). The new model independent GGSZ analysis [4] uses input from CLEO-c to determine $\gamma$ using $B^{ \pm} \rightarrow D K^{ \pm}$decays with both $D \rightarrow K_{S}^{0} \pi^{+} \pi^{-}$and $D \rightarrow K_{S}^{0} K^{+} K^{-}$. The analysis uses $2 \mathrm{fb}^{-1}$ of Run 2 data from $p p$ collisions at a centre of mass energy of $13 \mathrm{TeV}$ during 2015 and 2016. Two control samples are used, $B^{ \pm} \rightarrow D \pi^{ \pm}$with $D \rightarrow K_{S}^{0} \pi^{+} \pi^{-}$and $D \rightarrow K_{S}^{0} K^{+} K^{-}$and $B \rightarrow D^{* \pm} \mu^{\mp} v_{\mu} X$ with $D^{* \pm} \rightarrow D \pi^{ \pm}$and $D \rightarrow K_{S}^{0} \pi^{+} \pi^{-}$and $D \rightarrow K_{S}^{0} K^{+} K^{-}$, where $X$ indicates other particles that may have not been reconstructed.

The simultaneous fit to the invariant masses $m\left(D K^{ \pm}\right)$and $m\left(D \pi^{ \pm}\right)$is shown in Fig. 1, where the control mode is used to fix the number of misidentified $B^{ \pm} \rightarrow D \pi^{ \pm}$decays in the $B^{ \pm} \rightarrow D K^{ \pm}$ sample. The parameters are then fixed, and the fit is repeated in each of the bins shown in Fig. 2, where the yield $N$ in each bin is

$$
\begin{aligned}
& N_{ \pm i}^{+}=h_{B^{+}}\left(F_{\mp i}+\left(x_{+}^{2}+y_{+}^{2}\right) F_{ \pm i}+2 \sqrt{F_{i} F_{-i}}\left(x_{+} c_{ \pm i}+y_{+} s_{ \pm i}\right),\right. \\
& N_{ \pm i}^{-}=h_{B^{-}}\left(F_{ \pm i}+\left(x_{-}^{2}+y_{-}^{2}\right) F_{\mp i}+2 \sqrt{F_{i} F_{-i}}\left(x_{-} c_{ \pm i}+y_{-} s_{ \pm i}\right) .\right.
\end{aligned}
$$

Here $h_{B^{ \pm}}$are normalisation factors, $F_{i}$ give the fraction of $D^{0}$ and $\bar{D}^{0}$ decays in each bin of the Dalitz plot and come from the semileptonic control channel, $c_{i}$ and $s_{i}$ are the strong phase measurements from CLEO-c, and the $C P$ violation observables $x$ and $y$ are defined as

$$
x_{ \pm}=r_{B} \cos \left(\delta_{B} \pm \gamma\right), y_{ \pm}=r_{B} \sin \left(\delta_{B} \pm \gamma\right),
$$

with labels (+) for $B^{+}$and (-) $B^{-}$decays. The results for $x$ and $y$ are determined to be

$$
\begin{aligned}
& x_{+}=(-7.7 \pm 1.9 \pm 0.7 \pm 0.4) \times 10^{-2}, y_{+}=(-1.0 \pm 1.9 \pm 0.4 \pm 0.9) \times 10^{-2}, \\
& x_{-}=(9.0 \pm 1.7 \pm 0.7 \pm 0.4) \times 10^{-2}, y_{-}=(2.1 \pm 2.2 \pm 0.5 \pm 1.1) \times 10^{-2},
\end{aligned}
$$

which are combined with the results from Run 1 [5] to give $\gamma=\left(80_{-9}^{+10}\right)^{\circ}, r_{B}=0.080_{-0.011}^{+0.011}$ and $\delta_{B}=\left(110_{-10}^{+10}\right)^{\circ}$. This is the most precise single measurement of $\gamma$. 

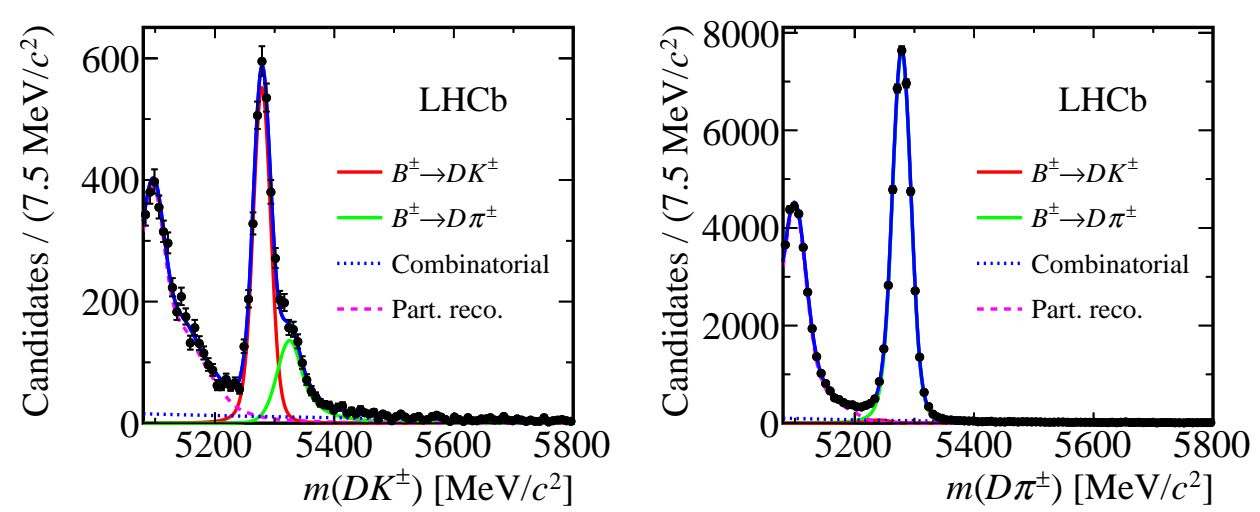

Figure 1: Simultaneous fit to the invariant $B$ candidate mass distribution for (left) $B^{ \pm} \rightarrow D K^{ \pm}$and (right) $B^{ \pm} \rightarrow D \pi^{ \pm}$decays. Figure reproduced from Ref. [4].
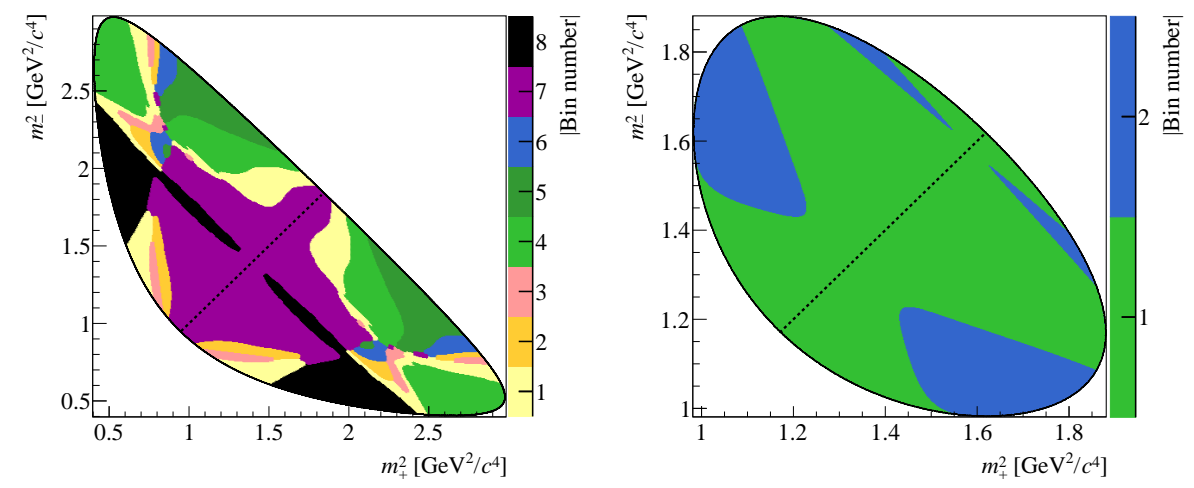

Figure 2: Dalitz plot binning scheme for (left) $D \rightarrow K_{S}^{0} \pi^{+} \pi^{-}$and (right) $D \rightarrow K_{S}^{0} K^{+} K^{-}$decays, reproduced from Ref. [4].

\section{The latest $\mathrm{LHCb} \gamma$ combination}

The best sensitivity to $\gamma$ comes from a combination of LHCb measurements, as described in Refs. [6] and [7]. This update includes three new results, which are described in detail in Refs. [4, $8,9]$. The combination follows the procedure defined in Ref. [6] and consists of 98 observables, from which 40 parameters are determined, including

$$
\gamma=\left(74.0_{-5.8}^{+5.0}\right)^{\circ}
$$

which is the world's most precise measurement from a single experiment, and is still dominated by the statistical uncertainty. The value from indirect measurements only is $\gamma=\left(65.6_{-3.4}^{+1.0}\right)^{\circ}$ [10] and is in better than $2 \sigma$ agreement with this combination. The consistency between the direct and indirect measurements of $\gamma$ is an important probe of new physics effects, so larger data samples are needed to reduce the uncertainty from direct determinations. Figure 3 (left) shows a breakdown of the combination by $B$ meson type, with consistency at the two sigma level. Note that only a single measurements drives the $B_{s}^{0}$ result. The evolution of $\gamma$ combination measurements from $\mathrm{LHCb}$ is shown in Fig. 3 (right). 

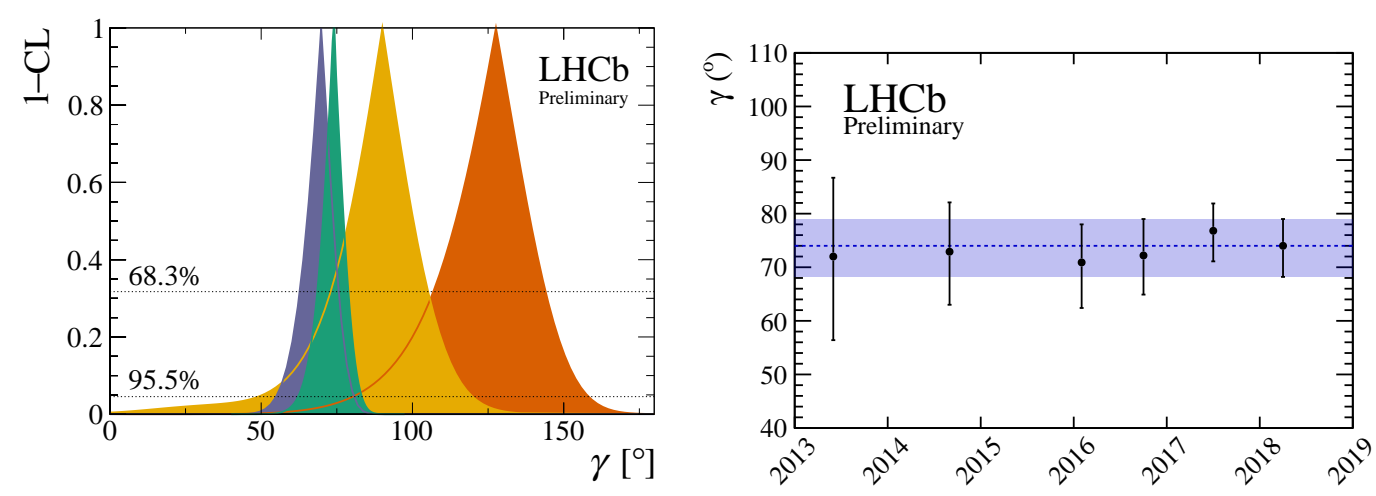

Figure 3: (Left) Comparison of $\gamma$ measurements using different types of $B$ mesons with $B^{+}$(blue), $B^{0}$ (yellow), $B_{s}^{0}$ (orange) and combined (green). (Right) The evolution of the LHCb $\gamma$ combination results. Reproduced from Ref. [7].

\section{Summary}

The worlds most precise single measurement of $\gamma$ using $B^{ \pm} \rightarrow D K^{ \pm}$decays with both $D \rightarrow$ $K_{S}^{0} \pi^{+} \pi^{-}$and $D \rightarrow K_{S}^{0} K^{+} K^{-}$was presented along with the most recent update of the LHCb $\gamma$ combination. In the future, the precision on $\gamma$ is expected to reach $4^{\circ}$ with Run 2 data and around $1^{\circ}$ after the LHCb upgrade, providing very precise tests of the Standard Model.

\section{References}

[1] Heavy Flavour Averaging Group, Averages of $b$-hadron, $c$-hadron, and $\tau$-lepton properties as of summer 2016, Eur. Phys. J. C77, 895 (2017).

[2] LHCb Collaboration, Measurement of the CKM angle $\gamma$ from a combination of $B \rightarrow D K$ results, LHCb-CONF-2017-004, 2017.

[3] A. Giri, Y. Grossman, A. Soffer and J. Zupan, Determining gamma using $B^{ \pm} \rightarrow D K^{ \pm}$with multibody D decays, Phys. Rev. D68 054018(2003).

[4] LHCb Collaboration, Measurement of the CKM angle $\gamma$ using $B^{ \pm} \rightarrow D K^{ \pm}$with $D \rightarrow K_{\mathrm{S}}^{0} \pi^{+} \pi^{-}$, JHEP 10 (2018) 107.

[5] LHCb Collaboration, Measurement of the CKM angle $\gamma$ using $B^{ \pm} \rightarrow D K^{ \pm}$with $D \rightarrow K_{\mathrm{S}}^{0} \pi^{+} \pi^{-}, K_{\mathrm{S}}^{0} K^{+} K^{-}$decays, JHEP 10 (2014) 097.

[6] LHCb Collaboration, Measurement of the CKM angle $\gamma$ from a combination of LHCb results, JHEP 12 (2016) 087.

[7] LHCb Collaboration, Update of the LHCb combination of the CKM angle $\gamma$, LHCb-CONF-2018-002, 2018.

[8] LHCb Collaboration, Measurement of $C P$ observables in $B^{ \pm} \rightarrow D K^{* \pm}$ decays using two- and four-body $D$ final states, JHEP 11 (2017) 156.

[9] LHCb Collaboration, Measurement of $C P$ violation in $B^{0} \rightarrow D^{\mp} \pi^{ \pm}$decays, JHEP 11 (2017) 156.

[10] CKMFitter Group, CP Violation and the CKM Matrix: Assessing the Impact of the Asymmetric B Factories, Eur. Phys. J. C41, 1-131 (2005). 